\title{
La percepción de los y las tituladas del itinerario dual en el grado de administración y dirección de empresas en el desarrollo de las competencias transversales de la titulación
}

\author{
José María Beraza Garmendia ${ }^{a}$ \\ ${ }^{a}$ Universidad del País Vasco UPV/EHU, josemaria.beraza@ehu.es
}

\begin{abstract}
One of the objectives of the European Higher Education Area is a change in teaching methodologies, transforming a teaching-focused education into an education focused on the learning process. Dual training has a place in this model, since it allows young people to obtain a degree, through a learning process that is developed in a shared way in the training school and in the company. The main objective of this work is to know the perception of the graduates of the Dual Itinerary in the Degree in Business Administration and Management on the development of transversal competences, in order to contrast whether these students actually acquire the skills established in the Degree Report to the same extent as the rest of the graduates of the Degree in Business Administration and Management. To this end, a survey of students participating in this Itinerary has been carried out. The results show that the most developed competencies and those that has most contributed in the Degree in Business Administration and Management are selflearning and teamwork. On the other hand, the skills with the least perceived development and to which the Degree in Business Administration and Management has contributed the least are foreign language communication and creativity, innovation and entrepreneurship.
\end{abstract}

Keywords: European Higher Education Area, Degree in Business Administration and Management, Dual Training, Transversal competencies.

\section{Resumen}

Uno de los objetivos del Espacio Europeo de Educación Superior es un cambio en las metodologías docentes, transformando una educación centrada en la enseñanza en una educación centrada en el proceso de aprendizaje. La formación dual tiene cabida en este modelo, puesto que posibilita a los jóvenes la obtención de un título, a través de un proceso de aprendizaje que se desarrolla de manera compartida en el centro de formación y en la empresa. El objetivo principal de este trabajo es conocer la percepción de los y las tituladas del Itinerario Dual en GADE sobre el desarrollo de las competencias transversales, para contrastar si realmente estos alumnos adquieren las competencias establecidas en la Memoria de la Titulación en igual medida que el resto de titulados y tituladas de GADE. Para ello se ha realizado una encuesta a alumnos/as participantes en este Itinerario. Los resultados muestran que las competencias más desarrolladas y a las que más ha contribuido el GADE son el aprendizaje autónomo y el trabajo en equipo. En cambio, las competencias con un menor desarrollo percibido y a las que menos ha contribuido el GADE son la comunicación en lengua extranjera y la creatividad, innovación y espiritu emprendedor. 
La percepción de los y las tiuladas del itinerario dual en el grado de administración y dirección de empresas en el desarrollo de las competencias transversales de la titulación

Palabras clave: Espacio europeo de educación superior, Grado en administración y dirección de empresas, Formación dual, Competencias transversales.

\section{Introducción}

La Universidad como Institución de Educación Superior es la fuente teórica generadora de conocimiento en los estudiantes. El ámbito donde el egresado de la universidad aplicará esos conocimientos es la empresa, que demanda conocimientos y competencias concretas acorde a los problemas que le surgen, y por extensión la sociedad. El punto de unión de ambas posturas que pueden parecer divergentes deben ser las habilidades integradoras de los conocimientos a las necesidades particulares de empresas y sociedad (Loades, 2005).

Uno de los objetivos fundamentales del Espacio Europeo de Educación Superior EEES es la búsqueda de un cambio de paradigma metodológico, tratando de transformar una educación centrada en la enseñanza por una educación centrada en el aprendizaje, donde el papel del alumno debería de tener un componente más activo (Villa y Poblete, 2007; Blanco, 2010).

En este sentido, el Real Decreto 1393/2007, de 29 de octubre, por el que se establece la ordenación de las enseñanzas universitarias oficiales conforme al Espacio Europeo de Educación Superior, impulsa un cambio en las metodologías docentes, que centra el objetivo en el proceso de aprendizaje del estudiante, en un contexto que se extiende ahora a lo largo de la vida, de manera que los planes de estudios conducentes a la obtención de un título deben tener en el centro de sus objetivos, por un lado, la adquisición de competencias por parte de los estudiantes, ampliando, sin excluir, el tradicional enfoque basado en contenidos y horas lectivas; $y$, por otro lado, hacer énfasis en los métodos de aprendizaje de dichas competencias así como en los procedimientos para evaluar su adquisición.

La formación dual tiene cabida en este modelo, puesto que posibilita a los jóvenes la obtención de un título, a través de un proceso de aprendizaje que se desarrolla de manera compartida en el centro de formación y en la empresa, que les permite desarrollar competencias complementarias (trabajo en equipo, responsabilidad,...), descubrir el funcionamiento diario de la empresa y adquirir conocimientos, destrezas y habilidades necesarias para ejercer una profesión y responder a las necesidades específicas de la empresa.

La formación dual se presenta como otra vía de formación para mejorar la empleabilidad y el desarrollo individual, incrementar la adecuación y la continuidad entre las exigencias del mundo profesional y la formación inicial de los estudiantes universitarios y conseguir una mayor eficiencia económica e integración social. Este tipo de enseñanza optimiza la profesionalización para un acceso directo al empleo (Arrieta, 2018; Corona y Sastre, 2018).

La formación dual universitaria ha sido implementada en muchos países europeos desde hace ya algunos años, pero son dos países los que han liderado este sistema de formación: Alemania, conocido con el nombre de formación dual, y Francia, con el nombre de formación en alternancia (Badia, 2014). En cambio, en España esta formación está dando todavía sus primeros pasos a nivel universitario.

En este contexto, a requerimiento del Equipo Rectoral del Campus de Gipuzkoa a la Dirección de la Sección de Gipuzkoa de la Facultad de Economía y Empresa (UPV/EHU), y con la colaboración de la Asociación de Empresarios de Gipuzkoa, ADEGI, se diseña e implanta la formación dual en el Grado en 
Administración y Dirección de Empresas, mediante el establecimiento de un minor o especialidad de "Formación Dual" 1 .

\section{Objetivos}

El objetivo principal de este trabajo consiste en conocer la percepción de los y las tituladas del Itinerario Dual en el Grado de Administración y Dirección de Empresas de la Facultad de Economía y Empresa de Donostia / San Sebastián, sobre el desarrollo de las competencias transversales, para contrastar si realmente han adquirido las competencias establecidas en la Memoria de la Titulación, en igual grado que el resto de los y las tituladas que no han cursado este Itinerario, con el fin último de, en su caso, mostrar que la formación dual es un proceso de enseñanza-aprendizaje válido para la efectiva adquisición de las mismas.

Además de este objetivo principal, la realización de este estudio también nos permitirá identificar si la realización del Itinerario Dual provoca un cambio en la percepción de la importancia de las competencias transversales establecidas en la Memoria de la Titulación, para su futuro desarrollo profesional.

Creemos que este estudio puede ser de interés no sólo para los Equipos Directivos de la titulación del Grado en Administración y Dirección de Empresas, sino que puede ser extensible también a los de otras titulaciones, en un momento en el que el Gobierno está planteándose la regulación de esta modalidad de formación ${ }^{2}$.

\section{Desarrollo de la innovación}

\subsection{Competencias transversales}

La memoria que configura el proyecto de un título oficial, que deben presentar las universidades para solicitar la verificación del mismo, debe incluir las competencias generales y específicas que los estudiantes deben adquirir durante sus estudios, que deben ser evaluables. El Real Decreto citado en el apartado anterior señala que se garantizarán, como mínimo las siguientes competencias básicas, en el caso del Grado:

- Que los estudiantes hayan demostrado poseer y comprender conocimientos en un área de estudio que parte de la base de la educación secundaria general, y se suele encontrar a un nivel que, si bien se apoya en libros de texto avanzados, incluye también algunos aspectos que implican conocimientos procedentes de la vanguardia de su campo de estudio;

- Que los estudiantes sepan aplicar sus conocimientos a su trabajo o vocación de una forma profesional y posean las competencias que suelen demostrarse por medio de la elaboración y defensa de argumentos y la resolución de problemas dentro de su área de estudio;

- Que los estudiantes tengan la capacidad de reunir e interpretar datos relevantes (normalmente dentro de su área de estudio) para emitir juicios que incluyan una reflexión sobre temas relevantes de índole social, científica o ética;

\footnotetext{
1 El diseño del Itinerario Dual por parte del Equipo de Dirección, su aprobación por la UPV/EHU, con la denominación de Formación Dual Universidad-Empresa, y el inicio de su implantación tuvo lugar a lo largo de 2017, previamente a la publicación por parte de la Agencia de calidad del Sistema Universitario Vasco (Unibasq) del Protocolo para la obtención del reconocimiento de formación dual para títulos universitarios oficiales de Grado y Máster. Dicho reconocimiento se logró, previa solicitud, en la primavera de 2018.

Tras la publicación del Protocolo, varias titulaciones ofertadas por las universidades del Sistema Universitario Vasco han obtenido el reconocimiento de formación dual.

${ }^{2}$ La Ley 11/2020 de 30 de diciembre de Presupuestos Generales del Estado para el año 2021 incorpora el contrato para la formación dual universitaria en el artículo 11 del Estatuto de los Trabajadores, que está pendiente de desarrollo reglamentario.
} 
- Que los estudiantes puedan transmitir información, ideas, problemas y soluciones a un público tanto especializado como no especializado;

- Que los estudiantes hayan desarrollado aquellas habilidades de aprendizaje necesarias para emprender estudios posteriores con un alto grado de autonomía.

Por su parte, la Memoria de la Titulación del Grado en Administración y Dirección de Empresas de la Facultad de Economía y Empresa incluye, además de las anteriores, las siguientes competencias transversales:

- Desarrollar las habilidades de aprendizaje para adquirir un alto grado de autonomía, tanto de cara a emprender estudios posteriores como de cara a su propia autoformación en un ámbito sujeto a continuos cambios e innovaciones.

- Capacidad para la comunicación escrita y oral con fluidez.

- Capacidad para trabajar en equipo, con responsabilidad y respeto, iniciativa y liderazgo.

- Capacidad para el pensamiento analítico y la reflexión crítica.

- Capacidad para comunicarse en una lengua extranjera, preferentemente inglés, francés o alemán.

Por tanto, en este trabajo se va a realizar el análisis de la percepción de los y las tituladas del Itinerario Dual en el Grado de Administración y Dirección de Empresas de la Facultad de Economía y Empresa de Donostia / San Sebastián, sobre el desarrollo de las competencias transversales que se acaban de describir.

\subsection{Itinerario Dual}

El Itinerario de Formación Dual: Universidad-Empresa se integra en el plan de estudios del Grado en Administración y Dirección de Empresas como un nuevo minor o especialidad, junto a los otros cuatro ya existentes en el grado que se imparte en la Sección de Gipuzkoa de la Facultad de Economía y Empresa de la UPV/EHU. Al igual que en estas especialidades, el itinerario dual se realiza durante el segundo cuatrimestre del tercer curso y el primer y segundo cuatrimestre del cuarto curso del Grado ${ }^{3}$.

En términos generales, el Itinerario de Formación Dual supone para el/la alumno/a, por un lado, la realización en el centro de dos asignaturas de orientación empresarial (10 ECTS) y, por otro lado, la estancia en una empresa diseñada en tres fases (33 ECTS). Por último, el/la alumno/a realiza y presenta un trabajo de fin de grado vinculado a su estancia en la empresa (12 ECTS). Por tanto, el Itinerario Dual supone una estancia en la empresa de 825 horas más las 300 correspondientes al TFG ${ }^{4}$.

La realización del itinerario dual, con la importante presencia en la empresa que supone, es totalmente compatible con los horarios de las asignaturas troncales que los/as alumnos/as deben cursar en tercer y cuarto curso.

El alumnado interesado en realizar este Itinerario Dual tiene que preinscribirse y seguir un proceso de selección, que se desarrolla en dos fases. La primera fase es la preselección por parte de la Facultad aplicando los siguientes criterios: número de créditos aprobados, nota media, currículo y carta de motivación en la que se expliquen las razones por las que se desea realizar la formación dual. En una segunda fase se realiza una entrevista personal para verificar que el candidato/a podrá adaptarse a los ritmos y a las exigencias de la formación en alternancia, y que posee las aptitudes necesarias para desarrollar las capacidades que le permitan un proceso de aprendizaje con buen desempeño en la empresa. La Facultad hace una preselección de 2/3 alumnos/as por cada oferta de empresa, y la empresa selecciona

\footnotetext{
${ }^{3}$ Para un conocimiento más detallado de las características de este Itinerario Dual puede consultarse (Beraza y Azkue, 2018).

${ }^{4}$ La adaptación del Itinerario al Protocolo anteriormente citado ha supuesto algunos cambios en su diseño, entre los que cabe destacar el incremento de la estancia en la empresa hasta los 38 ECTS.
} 
al candidato en una siguiente fase, ya que la mayoría de las empresas reconocen la necesidad de poder ser ellas quienes elijan al alumno que participará en el programa de formación dual realizando la estancia en su empresa.

En la primera promoción ${ }^{5}$ se inscribieron un total de 38 alumnos/as y tras la fase de entrevistas personales, la Facultad hizo una preselección de 20 alumnos/as a presentar a las empresas.

Por su parte, tras la campaña de captación de empresas, 20 empresas mostraron interés en participar, pero finalmente fueron 7 las empresas seleccionadas para participar en el Itinerario, tres de las cuales estaban dispuestas a acoger a dos estudiantes.

Finalmente, fueron 10 los estudiantes seleccionados para participar en el Itinerario, y que lo han finalizado, con la excepción de una alumna que, a los pocos meses de iniciar la estancia en la empresa, decidió renunciar por motivos personales a continuar en el Itinerario.

A través de una encuesta se recogió la opinión de los tres principales agentes intervinientes: alumnado, tutores/as académicos e instructores/as de empresa. La valoración general de los tres es muy positiva.

\subsection{Metodología}

Este estudio, cuya ficha técnica se recoge en la tabla 1, tiene un carácter descriptivo, utilizando como instrumento de recogida de información una encuesta autocumplimentada enviada por correo electrónico y dirigida a los/as alumnos/as, que han participado en el proceso de selección de la primera promoción de este Itinerario Dual.

La encuesta ha sido pasada a la finalización del programa formativo del Itinerario Dual en el curso 18-19.

El cuestionario empleado es el mismo que se utilizó en un trabajo anterior para conocer la percepción de los y las tituladas en el Grado de Administración y Dirección de Empresas de la Facultad de Economía y Empresa de Donostia / San Sebastián, sobre el desarrollo de las competencias transversales ${ }^{6}$.

\section{Tabla 1. Ficha técnica del estudio empírico}

\begin{tabular}{ll}
\hline Universo/Población & $\begin{array}{l}\text { Alumnado participante en el proceso de selección de la primera promoción } \\
\text { del Itinerario Dual del Grado en ADE en la Facultad de Economía y } \\
\text { Empresa. Sección Donostia / San Sebastián en el curso. }\end{array}$ \\
$\begin{array}{l}\text { Tamaño de la población } \\
\text { Método de recogida de la } \\
\text { información }\end{array}$ & $\begin{array}{l}\text { Encuesta on line con encuesta fácil con } 77 \text { cuestiones cerradas. } \\
\text { Muestra Total }\end{array}$ \\
$\begin{array}{l}\text { Tasa de respuesta } \\
\text { Fecha de realización }\end{array}$ & $\begin{array}{l}100 \% . \\
\text { Técnicas de análisis }\end{array}$ \\
\hline
\end{tabular}

Fuente: Elaboración propia.

El cuestionario se estructura en cuatro bloques. En el primero, y utilizando una escala multi ítem de Likert de cinco puntos para cada competencia, se solicita al alumnado participante en el proceso de selección de la primera promoción del Itinerario Dual que indique el nivel de desarrollo alcanzado en cada una de ellas.

\footnotetext{
${ }^{5}$ Para un conocimiento más detallado de la primera promoción puede consultarse (Beraza, Azkue y Villalba, 2020).

${ }^{6}$ Para un conocimiento más detallado de los resultados de este estudio puede consultarse (Beraza y Villalba, 2017).
} 
En el segundo y tercer bloque se solicita al encuestado/a su opinión respecto al grado de desarrollo de las mismas atribuible a sus estudios de ADE y el grado de importancia que tienen esas competencias para su futuro profesional, para lo que se utiliza, solamente, la definición de cada competencia.

El cuarto y último bloque recoge datos de clasificación como edad, sexo, fecha de inicio de los estudios, realización de prácticas, participación en programas de movilidad y experiencia profesional.

Con los datos obtenidos se han realizado análisis de tipo descriptivo: frecuencias y media. Para todos estos análisis se ha utilizado el programa estadístico SPSS.

\section{Resultados}

\subsection{Perfil de la muestra}

Los encuestados tienen entre 21 y 22 años. Teniendo en cuenta que la edad media de ingreso a la universidad es de 18 años, parece lógico que tengan esta edad.

La mayoría del alumnado participante es mujer, en concreto un $66,7 \%$. Un porcentaje algo superior al de la proporción de mujeres de nuevo ingreso en el Grado, que está en torno al 50\%.

Salvo dos casos, el resto no había realizado prácticas durante los estudios antes de iniciar el Itinerario Dual y sólo en un caso se había participado en un programa de movilidad. Estos resultados son lógicos, ya que hay que tener en cuenta que tanto las prácticas como la movilidad se realizan a partir del tercer curso. Solo una alumna tenía alguna experiencia profesional relacionada con los estudios de GADE en esa fecha. No obstante, una alumna asignada al Itinerario Dual ha realizado prácticas y movilidad, y tiene experiencia profesional relacionada con los estudios.

\subsection{Percepción de las competencias analizadas}

El análisis descriptivo comienza con la autopercepción que tienen, las y los encuestados, sobre el grado de consecución de las competencias transversales, en el desarrollo de estas competencias en sus estudios del Grado y en la importancia que les atribuyen para su futuro profesional a la finalización del Itinerario Dual en junio de 2019. En un segundo bloque se comparan las diferencias existentes con el grado de desarrollo y el grado de importancia.

Respecto al grado de consecución de las competencias transversales, esta información se ha obtenido a través del planteamiento de diez situaciones a partir de las cuales los encuestados indicaban su grado de acuerdo o desacuerdo, por medio de una escala de Likert de 5 puntos, a las diferentes dimensiones que constituían cada competencia analizada.

En las siguientes tablas se recoge el porcentaje de la muestra que se muestra de acuerdo o muy de acuerdo con los diferentes ítems (G.A.) y la media. 
Tabla 2. Desarrollar las habilidades de aprendizaje para adquirir un alto grado de autonomía

\begin{tabular}{lcc}
\hline Cuando me enfrento a un proceso o tarea de aprendizaje... & $\mathbf{2 0 1 9}$ \\
\hline Me marco unos objetivos a alcanzar. & \% G.A. Media \\
Planifico y organizo mi propio trabajo para asegurar los resultados. & 77,8 & 3,89 \\
Con mi esfuerzo individual consigo los objetivos que me propongo. & 100 & 4,33 \\
Planifico o gestiono el tiempo necesario para lograr lo que me propongo. & 88,9 & 4,56 \\
Cumplo con la planificación que he realizado. & 77,8 & 4,33 \\
Soy capaz de utilizar la información recomendada para la correcta ejecución de mis & 88,9 & 4,22 \\
tareas. & 88,9 & 4,11 \\
Soy constante en mi trabajo a pesar de los obstáculos que se presenten. & 100 & 4,78 \\
\hline
\end{tabular}

Fuente: Elaboración propia.

Las situaciones planteadas en el cuestionario se presentan en los títulos de las tablas que recogen los resultados obtenidos para cada competencia.

La mayoría de los encuestados muestran su acuerdo con las afirmaciones planteadas obteniendo valoraciones medias superiores a 4, excepto el marcarse unos objetivos a alcanzar. La mayor valoración la alcanzan la constancia en el trabajo y el esfuerzo individual (tabla 2).

En conclusión, el alumnado se ve capacitado para desenvolverse, de forma individual, en cualquier proceso o tarea de aprendizaje, tal como se refleja en los altos porcentajes, de acuerdo, obtenidos en casi todos los ítems.

A diferencia de la pregunta anterior, las opciones más elegidas han sido la 3 y la 4 por lo que la valoración media, aunque todas están por encima del 3, es sensiblemente inferior. Además, el grado de acuerdo con los diferentes ítems, en ningún caso, alcanza el 70\% (tabla 3). Por tanto, el alumnado también se ve capacitado, aunque en menor medida, para aplicar los conocimientos adquiridos al trabajo y tomar decisiones fundadas.

Tabla 3. Aplicar los conocimientos adquiridos al trabajo (y tomar decisiones) en cualquier campo relacionado con la administración y dirección de empresas

\begin{tabular}{|c|c|c|}
\hline \multirow{2}{*}{$\begin{array}{l}\text { Cuando tengo que tomar una decisión o buscar una solución a un problema (de } \\
\text { gestión empresarial)... }\end{array}$} & \multicolumn{2}{|c|}{2019} \\
\hline & $\%$ G.A. & Media \\
\hline $\begin{array}{l}\text { Identifico correctamente la naturaleza del mismo y soy capaz de descomponerlo en } \\
\text { partes manejables. }\end{array}$ & 77,8 & 3,78 \\
\hline $\begin{array}{l}\text { Aplico sistemáticamente un método de análisis que me permite identificar las causas } \\
\text { del mismo y evaluar su impacto. }\end{array}$ & 44,4 & 3,22 \\
\hline Aplico correctamente conocimientos teóricos a la resolución del mismo. & 66,7 & 3,56 \\
\hline Presento más de una alternativa de solución del mismo. & 33,3 & 3,22 \\
\hline Propongo la solución más adecuada. & 55,5 & 3,67 \\
\hline $\begin{array}{l}\text { Si la situación lo requiere no tengo dificultad en aplicar técnicas matemáticas y } \\
\text { estadísticas para su resolución. }\end{array}$ & 66,6 & 3,78 \\
\hline $\begin{array}{l}\text { Si la situación lo requiere no tengo dificultad en utilizar tecnologías informáticas de } \\
\text { gestión empresarial para su resolución. }\end{array}$ & 66,7 & 3,78 \\
\hline
\end{tabular}

Fuente: Elaboración propia. 

empresas en el desarrollo de las competencias transversales de la titulación

La percepción del alumnado respecto a su capacidad de trabajar en equipo parece bastante generalizada a excepción de la dirección de reuniones que fomenten la participación y compromiso del equipo (tabla 4).

A la vista de los resultados, la creatividad, innovación y espíritu emprendedor es una competencia no muy desarrollada. Salvo su actitud receptiva a nuevas ideas que se le presenten, el resto de los ítems tienen una valoración inferior a 4. Destaca, negativamente, su limitación a la hora de hacer propuestas originales y poco convencionales. (tabla 5).

Aunque los/as encuestados/as no tienen dificultad en identificar y clasificar los elementos principales a la hora de analizar una situación empresarial, en establecer relaciones entre los mismos, y son capaces de formarse su propia opinión teniendo en cuenta la opinión de los demás, no se trata de una competencia demasiado desarrollada. Basarse en conocimientos teóricos adecuados y saber a qué fuentes recurrir cuando falta información o hay incoherencia de los datos son los ítems con menor valoración (tablas 6 y 7 ).

Tabla 4. Trabajar en equipo, con responsabilidad y respeto, iniciativa y liderazgo

\begin{tabular}{|c|c|c|}
\hline \multirow[t]{2}{*}{ A la hora de trabajar en equipo... } & \multicolumn{2}{|c|}{2019} \\
\hline & $\%$ G.A & Media \\
\hline Colaboro en la definición, organización y distribución de las tareas de grupo. & 88,9 & 4,00 \\
\hline Cumplo con las tareas que se me han asignado en el equipo. & 100 & 4,78 \\
\hline $\begin{array}{l}\text { Tengo una participación activa (comparto información y experiencias) en las } \\
\text { reuniones del equipo. }\end{array}$ & 100 & 4,33 \\
\hline $\begin{array}{l}\text { Colaboro en la planificación (organización y distribución de tareas) del trabajo en } \\
\text { equipo. }\end{array}$ & 77,8 & 4,00 \\
\hline $\begin{array}{l}\text { Tengo en cuenta los puntos de vista de los demás e interacciono de forma } \\
\text { constructiva. }\end{array}$ & 88,9 & 4,11 \\
\hline $\begin{array}{l}\text { Dirijo las reuniones del grupo consiguiendo la participación y compromiso de todos } \\
\text { los miembros del grupo. }\end{array}$ & 33,3 & 3,22 \\
\hline
\end{tabular}

Fuente: Elaboración propia.

Tabla 5. Creatividad, innovación y espíritu emprendedor

\begin{tabular}{|c|c|c|}
\hline \multirow{3}{*}{$\begin{array}{l}\text { Personalmente considero que... } \\
\text { Frente a un problema empresarial, tengo facilidad para generar ideas o soluciones } \\
\text { nuevas. }\end{array}$} & \multicolumn{2}{|c|}{2019} \\
\hline & \multicolumn{2}{|c|}{$\%$ G.A. Media } \\
\hline & 55,6 & 3,56 \\
\hline Soy capaz de analizar los riesgos y beneficios de las ideas que propongo. & 66,7 & 3,67 \\
\hline Tengo una actitud abierta ante nuevas ideas y perspectivas. & 100 & 4,78 \\
\hline Las ideas que propongo suelen ser originales o poco convencionales. & 22,2 & 3,33 \\
\hline $\begin{array}{l}\text { Soy capaz de integrar conocimientos de diferentes materias para generar ideas } \\
\text { novedosas a problemas y situaciones conocidas o desconocidas dentro del mundo } \\
\text { empresarial. }\end{array}$ & 44,4 & 3,44 \\
\hline Soy capaz de implicar a los demás en las propuestas que realizo. & 77,8 & 3,89 \\
\hline $\begin{array}{l}\text { Tengo una visión de futuro que me permite identificar nuevas oportunidades de } \\
\text { negocio. }\end{array}$ & 44,4 & 3,44 \\
\hline Me siento capaz de llevar a la práctica mis ideas (planes, proyectos, etc.). & 44,4 & 3,56 \\
\hline
\end{tabular}

Fuente: Elaboración propia. 
Tabla 6. Pensamiento analítico y reflexión crítica (I)

Si he de analizar una determinada situación empresarial...

2019

\% G.A. Media
$77,8 \quad 3,89$
$66,6 \quad 4,00$
conclusiones.

Si detecto que falta información o hay incoherencias en los datos disponibles, sé a qué fuentes de información debo recurrir para completarla o resolverla.

Tabla 7. Pensamiento analítico y reflexión crítica (II)

Cuando doy mi opinión sobre una situación empresarial...

$\begin{array}{cr}\text { \% G.A. Media } \\ 77,8 & 4,00 \\ 66,7 & 3,67 \\ 77,8 & 4,33\end{array}$

Fuente: Elaboración propia.

La emisión de juicios razonados basándose en los datos obtenidos no supone un problema para la mayoría de los encuestados, pero no se trata de una competencia demasiado desarrollada. Ninguno de los los 4 ítems alcanza una valoración de 4 (tabla 8).

Tabla 8. Emitir juicios razonados apoyándose en los datos obtenidos

\begin{tabular}{|c|c|c|}
\hline Cuando tengo que emitir un juicio razonado... & \multicolumn{2}{|c|}{2019} \\
\hline & \multicolumn{2}{|c|}{ \% G.A. Media } \\
\hline $\begin{array}{l}\text { Selecciono los datos adecuados para fundamentar mis interpretaciones y } \\
\text { conclusiones. }\end{array}$ & 66,6 & 3,89 \\
\hline $\begin{array}{l}\text { Con la información de que dispongo soy capaz de extraer } \\
\text { conclusiones/implicaciones. }\end{array}$ & 77,8 & 3,89 \\
\hline $\begin{array}{l}\text { Sé perfectamente cuándo una afirmación es una opinión y cuándo es un hecho } \\
\text { constatado. }\end{array}$ & 66,6 & 3,89 \\
\hline Lo hago apoyándome en datos o evidencias válidas. & 66,6 & 3,89 \\
\hline
\end{tabular}

Fuente: Elaboración propia.

A la vista de los resultados, la comunicación oral es una competencia no muy desarrollada. Salvo la capacidad para expresarse oralmente de forma clara y ordenada y la capacidad para encontrar y utilizar buenos ejemplos para facilitar la comprensión de una idea, el resto de los ítems no alcanzan el 50\% de grado de acuerdo. Destaca, negativamente, su limitación a hablar de forma segura y con naturalidad. (tabla 9). 
La percepción de los y las tiuladas del itinerario dual en el grado de administración y dirección de empresas en el desarrollo de las competencias transversales de la titulación

Tabla 9. Comunicación oral con fluidez

\begin{tabular}{|c|c|c|}
\hline A la hora de hablar... & \multicolumn{2}{|c|}{2019} \\
\hline & \multicolumn{2}{|c|}{ \% G.A. Media } \\
\hline Cuando me expreso oralmente, lo hago de forma clara y ordenada. & 55,6 & 3,56 \\
\hline Cuando hablo en público, me muestro seguro e intervengo con naturalidad. & 33,3 & 3,22 \\
\hline $\begin{array}{l}\text { En mis intervenciones orales, controlo y manejo mi lenguaje no verbal (gestos, } \\
\text { expresión, volumen de voz, contacto visual, etc.), adecuándolo a las circunstancias y } \\
\text { objetivos de cada situación. }\end{array}$ & 44,4 & 3,56 \\
\hline $\begin{array}{l}\text { Cuando tengo que comunicar una idea, no tengo dificultad en encontrar y utilizar } \\
\text { buenos ejemplos para facilitar su comprensión. }\end{array}$ & 55,6 & 3,44 \\
\hline $\begin{array}{l}\text { Si hago una exposición oral, me muestro abierto al diálogo (no me importa tener que } \\
\text { responder a preguntas que me hagan). }\end{array}$ & 44,4 & 3,44 \\
\hline
\end{tabular}

Fuente: Elaboración propia.

La competencia de comunicación escrita es una competencia asumida por un porcentaje de la muestra mayor, por lo que podemos deducir que nuestros egresados consideran que se comunican de una manera más efectiva por escrito, salvo la utilización de citas y referencias bibliográficas, lo cual sorprende un poco dada la cercanía de la encuesta con la realización del Trabajo Fin de Grado (tabla 10).

Tabla 10. Comunicación escrita con fluidez.

\begin{tabular}{lcc}
\hline A la hora de escribir... & $\mathbf{2 0 1 9}$ \\
\hline & \% G.A. Media \\
$\begin{array}{l}\text { Me expreso por escrito con total corrección gramatical (frases bien ordenadas y } \\
\text { estructuradas, ortografía, puntos, comas...). }\end{array}$ & 66,7 & 4,33 \\
$\begin{array}{l}\text { Al escribir, manejo adecuadamente el lenguaje técnico necesario. } \\
\begin{array}{l}\text { Cuando tengo que redactar un texto, lo hago de forma clara, ordenada y } \\
\text { comprensible (relacionando con lógica ideas, argumentos y conclusiones). }\end{array}\end{array}$ & 66,6 & 4,00 \\
$\begin{array}{l}\text { A la hora de expresarme por escrito, soy capaz de hacerlo utilizando mis propias } \\
\text { palabras, consiguiendo que el texto se entienda bien. }\end{array}$ & 88,9 & 3,89 \\
$\begin{array}{l}\text { Cuando tengo que redactar un texto, sé en qué ocasiones debo de remitirme a citas y } \\
\text { referencias bibliográficas, y de qué forma he de hacerlo. }\end{array}$ & 44,4 & 3,33
\end{tabular}

Fuente: Elaboración propia.

A la vista de los resultados, muchos de los egresados manifiestan problemas a la hora de comunicarse en un idioma extranjero. Esta carencia es más manifiesta cuando tienen que transmitir información que cuando tienen que recibirla. Cabe destacar también que menos del $40 \%$ de los encuestados se considera capaz de mantener una conversación fluida o realizar presentaciones orales en una lengua extranjera (tabla 11). 
Tabla 11. Comunicarse en una lengua extranjera con fluidez

En un entorno de comunicación en un idioma extranjero...

$\%$ G.A. Media

Si asisto a una conferencia, un debate o veo la Tv, comprendo las ideas principales (sigo el hilo, el argumento).

$77,7 \quad 4,11$

Soy capaz de leer textos complejos (artículos, informes técnicos, etc.), entendiendo las principales ideas.

Puedo mantener una conversación fluida con hablantes nativos, debatir, defender mis ideas, etc.

Soy capaz de hacer descripciones y presentaciones orales claras y detalladas acerca de cuestiones cotidianas o relativas a mi ámbito de estudio.

Escribo de forma correcta, clara y estructurada: redacciones, informes, cartas, etc.

Fuente: Elaboración propia.

Finalmente, en función de las valoraciones medias, las competencias analizadas se pueden ordenar, de mayor a menor valor medio, tal como muestra la tabla 12. Las competencias más desarrolladas, con una media superior a 4, son: el aprendizaje autónomo y el trabajo en equipo. El resto de las competencias obtienen una media entre 3,5 y 4, excepto la comunicación oral, que queda ligeramente por debajo.

Una vez que los encuestados manifestaron su nivel de desarrollo para cada una de las competencias, se les preguntó por la contribución que los estudios en ADE habían tenido en el mismo. Para ello se les pidió, a través de una escala de Likert de cinco puntos, que iba desde "nula contribución" a máxima contribución, que valorasen dicha contribución, obteniéndose los resultados de la tabla 13. Las competencias a cuyo desarrollo más ha contribuido el GADE en el período analizado son: el aprendizaje autónomo, el trabajo en equipo y la emisión de juicios razonados. Por el contrario, las competencias a cuyo desarrollo menos contribuye el GADE son: la comunicación en lengua extranjera y la creatividad, innovación y espíritu emprendedor.

Tabla 12. Desarrollo percibido por parte de los encuestados

\begin{tabular}{lccc}
\hline Competencia & \multicolumn{2}{c}{$\mathbf{2 0 1 9}$} \\
\hline & \multicolumn{2}{c}{ Media } & Orden \\
Aprendizaje autónomo & 4,32 & $1^{\circ}$ \\
Trabajo en equipo & 4,07 & $2^{\circ}$ \\
Pensamiento analítico & 3,91 & $3^{\circ}$ \\
Emitir juicios razonados & 3,89 & $4^{\circ}$ \\
Comunicación escrita con fluidez & 3,87 & $5^{\circ}$ \\
Creatividad, innovación y espíritu emprendedor. & 3,71 & $6^{\circ}$ \\
Comunicación en lengua extranjera & 3,71 & $7^{\circ}$ \\
Aplicar los conocimientos adquiridos & 3,57 & $8^{\circ}$ \\
Comunicación oral & 3,44 & $9^{\circ}$ \\
\hline
\end{tabular}


La percepción de los y las tiuladas del itinerario dual en el grado de administración y dirección de empresas en el desarrollo de las competencias transversales de la titulación

Tabla 13. Contribución de los estudios de GADE

\begin{tabular}{lcc}
\hline Competencia & \multicolumn{2}{c}{2019} \\
\hline & Media & Orden \\
Aprendizaje autónomo & 4,00 & $1^{\circ}$ \\
Trabajo en equipo & 4,00 & $2^{\circ}$ \\
Emitir juicios razonados & 4,00 & $3^{\circ}$ \\
Pensamiento analítico & 3,89 & $4^{\circ}$ \\
Comunicación escrita con fluidez & 3,67 & $5^{\circ}$ \\
Aplicar los conocimientos adquiridos & 3,67 & $6^{\circ}$ \\
Comunicación oral & 3,56 & $7^{\circ}$ \\
Creatividad, innovación y espíritu emprendedor & 2,78 & $8^{\circ}$ \\
Comunicación en lengua extranjera & 2,67 & $9^{\circ}$
\end{tabular}

Fuente: Elaboración propia.

A continuación, se pidió a los encuestados que manifestaran su opinión respecto al grado de importancia de las competencias transversales analizadas en su futuro profesional. Para ello se utilizó una escala de Likert de cinco puntos que iba desde "nada importante" a "muy importante". La tabla 14 recoge la puntuación media, así como el orden de importancia, para cada competencia.

Cabe destacar que todas las competencias tienen una puntuación superior a 4, lo que significa que los encuestados consideran importantes todas las competencias para su futuro profesional. Llama la atención que la competencia que tiene más importancia para los encuestados para su futuro profesional es por unanimidad la comunicación escrita con fluidez y la que menos la creatividad, innovación y espíritu emprendedor.

Tabla 14. Grado de importancia para el futuro profesional

\begin{tabular}{lcc}
\hline Competencia & \multicolumn{2}{c}{$\mathbf{2 0 1 9}$} \\
\hline & Media & Orden \\
Comunicación escrita con fluidez & 5,00 & $1^{\circ}$ \\
Comunicación oral & 4,78 & $2^{\circ}$ \\
Trabajo en equipo & 4,78 & $3^{\circ}$ \\
Emitir juicios razonados & 4,78 & $4^{\circ}$ \\
Aprendizaje autónomo & 4,67 & $5^{\circ}$ \\
Pensamiento analítico & 4,56 & $6^{\circ}$ \\
Aplicar los conocimientos adquiridos & 4,56 & $7^{\circ}$ \\
Comunicación en lengua extranjera & 4,33 & $8^{\circ}$ \\
Creatividad, innovación y espíritu emprendedor & 4,11 & $9^{\circ}$ \\
\hline
\end{tabular}

Fuente: Elaboración propia.

(cc) EY-NC-ND 2021, Universitat Politècnica de València

Congreso In-Red (2021) 
Tabla 15. Comparación entre Grado de importancia, Desarrollo percibido y Contribución GADE

\begin{tabular}{|c|c|c|c|c|c|c|}
\hline Competencia & GI & DP & GI-DP & CG & DP-CG & GI-CG \\
\hline Comunicación escrita con fluidez & 5,00 & 3,87 & 1,13 & 3,67 & 0,2 & 1,33 \\
\hline Comunicación oral & 4,78 & 3,44 & 1,34 & 3,56 & $-0,12$ & 1,22 \\
\hline Trabajo en equipo & 4,78 & 4,07 & 0,71 & 4 & 0,07 & 0,78 \\
\hline Emitir juicios razonados & 4,78 & 3,89 & 0,89 & 4 & $-0,11$ & 0,78 \\
\hline Aprendizaje autónomo & 4,67 & 4,32 & 0,35 & 4 & 0,32 & 0,67 \\
\hline Pensamiento analítico & 4,56 & 3,91 & 0,65 & 3,89 & 0,02 & 0,67 \\
\hline Aplicar los conocimientos adquiridos & 4,56 & 3,57 & 0,99 & 3,67 & $-0,1$ & 0,89 \\
\hline Comunicación en lengua extranjera & 4,33 & 3,71 & 0,62 & 2,67 & 1,04 & 1,66 \\
\hline $\begin{array}{l}\text { Creatividad, innovación y espíritu } \\
\text { emprendedor }\end{array}$ & 4,11 & 3,71 & 0,4 & 2,78 & 0,77 & 1,32 \\
\hline
\end{tabular}

Fuente: Elaboración propia.

Con el objetivo de identificar aquellas competencias con un mayor desajuste entre las tres dimensiones analizadas: grado de importancia, desarrollo percibido y la contribución de los estudios de ADE, se procedió a calcular las diferencias entre los valores medios obtenidos por cada una de ellas, obteniendo los resultados que se recogen en la tabla 15. Se han destacado en rojo las diferencias superiores a 0,5. Como se puede apreciar existen diferencias superiores a 0,5 entre el grado de importancia y el desarrollo percibido por los encuestados en todas las competencias menos en el aprendizaje autónomo. Destacan con diferencias superiores a 1: comunicación oral y comunicación escrita con fluidez, por este orden. A su vez existen diferencias superiores a 0,5 entre el desarrollo percibido y el grado de contribución de GADE a su consecución en dos competencias: comunicación en lengua extranjera y creatividad, innovación y espíritu emprendedor. Finalmente, cabe destacar que existen diferencias superiores a 0,5 entre el grado de importancia y el grado de contribución de GADE a su consecución en todas las competencias, destacando las diferencias en cuatro competencias, en concreto: comunicación en lengua extranjera, comunicación escrita con fluidez, creatividad, innovación y espíritu emprendedor y comunicación oral.

\section{Conclusiones}

Teniendo en cuenta que los resultados obtenidos tienen que interpretarse con cautela en la medida en que se basan en las percepciones del alumnado que ha cursado el Itinerario Dual, además de la limitación del tamaño de la muestra utilizada, las principales conclusiones de este primer análisis son las siguientes:

Todas las competencias parecen tener un grado de importancia elevado, si bien el grado de desarrollo alcanzado de todas las competencias es menor que su grado de importancia.

Las competencias más desarrolladas son las de "aprendizaje autónomo" y "trabajo en equipo". En cambio, las competencias con un menor desarrollo percibido son las de "comunicación oral", "aplicar los conocimientos adquiridos", "comunicación en lengua extranjera" y "creatividad, innovación y espíritu emprendedor".

Las competencias a cuyo desarrollo más ha contribuido el GADE son: el aprendizaje autónomo, el trabajo en equipo y la emisión de juicios razonados. Por el contrario, las competencias a las que menos 
contribuyen los estudios de GADE son, por diferencia, las de "comunicación en lengua extranjera" y "creatividad, innovación y espíritu emprendedor", y, en menor medida, "comunicación oral" y "aplicar los conocimientos adquiridos".

Sorprende que después de curso y medio de formación dual no se hayan desarrollado en mayor medida la comunicación oral y la aplicación de los conocimientos adquiridos. Quizás podría ser debido a que el tipo de puestos y tareas desempeñadas en las empresas, no requieran desarrollar especialmente la comunicación oral ni una aplicación sistemática de los conocimientos adquiridos en la toma de decisiones y en la resolución de problemas.

Por el contrario, la menor contribución de GADE a la comunicación en lengua extranjera y la creatividad, innovación y espíritu emprendedor entra dentro de lo esperado. Por un lado, la realización del Itinerario Dual, aunque no impide, dificulta la participación en programas de movilidad (Erasmus,...) y es incompatible con la realización de asignaturas optativas de idiomas extranjeros que se ofrecen en la titulación. Por otro, el perfil profesional que demandan las empresas participantes en el Itinerario Dual no requiere de creatividad, innovación y espíritu emprendedor. Además, otros estudios internos realizados al alumnado de la titulación muestran que esta es la competencia que menos se desarrolla en el Grado.

Esto queda confirmado al observar que las competencias a las que menos contribuyen los estudios de GADE en relación al desarrollo percibido son "comunicación en lengua extranjera" y "creatividad, innovación y espíritu emprendedor".

Por su parte, las competencias a las que menos contribuyen los estudios de GADE en relación a su grado de importancia son "comunicación en lengua extranjera", "comunicación escrita con fluidez", "creatividad, innovación y espíritu emprendedor" y "comunicación oral”.

Del análisis anterior parece deducirse la necesidad de impulsar el desarrollo, en mayor medida, de las competencias de comunicación, en general, la creatividad, innovación y espíritu emprendedor, y, en menor medida, la aplicación de los conocimientos adquiridos al trabajo y a la toma de decisiones.

La flexibilización de las condiciones para facilitar la participación en programas de movilidad del alumnado del Itinerario Dual permitiría mejorar dos de estas competencias: "comunicación oral" y “comunicación en lengua extranjera". También se podría establecer que uno de los requisitos para optar al Itinerario Dual fuera disponer de un cierto conocimiento de idiomas extranjeros.

La comunicación oral y escrita se podrían desarrollar en las asignaturas de Orientación a la Empresa dentro del propio Itinerario.

Más difícil resulta impulsar en el marco del Itinerario Dual el desarrollo de la competencia de "creatividad, innovación y espíritu emprendedor".

Uno de los motivos para la implantación de este Itinerario fue el desarrollo de la competencia de "aplicar los conocimientos adquiridos", que a la vista de estos primeros resultados no parece haberse logrado. La consolidación del Itinerario con la oferta de perfiles profesionales más acordes con las competencias de la titulación podrían solventar este problema. 


\section{Referencias}

AA. VV. (2010). Memoria verificada del título oficial de Grado en Administración y Dirección de Empresas de la Facultad de Economía y Empresa (Sección Gipuzkoa). Universidad del País Vasco: San Sebastián.

Arrieta, F.J. (2018). "La formación dual en el ámbito universitario como alternativa a los retos planteados por la industria 4.0 en cuanto a la empleabilidad de los jóvenes" en Villalba, A. (Coord.). La revolución tecnológica y sus efectos en el mercado de trabajo: un reto del Siglo XXI. Madrid: Wolters Kluwer.

Badia, F. (Coord.) (2014). Promoció i desenvolupament de la formació dual en el sistema universitari català. Barcelona: Associació Catalana d'Universitats Públiques.

Beraza, J.M. y Azkue, I. (2018). "Diseño de un Itinerario de Formación Dual: Universidad-Empresa en GADE” en Journal of Management and Business Education, 1, 1, 53-68.

Beraza, J.M., Azkue, I., y Villalba, F.J. (2020). "Valoración del Itinerario de Formación Dual en el Grado en Administración y Dirección de Empresas". En Hernández, A.B. y Barberà, M.G. (Eds.): Gestión Empresarial y Objetivos de Desarrollo Sostenible (XXXIV Congreso Anual AEDEM). Reus: European Academic Publisher.

Beraza, J.M. y Villalba, F.J. (2017). "La percepción de los y las tituladas en el Grado en Administración y Dirección de Empresas en el desarrollo de las competencias transversales" en Revista de Dirección y Administración de Empresas, 24, 135-154.

Blanco, A. (coord.) (2010). Desarrollo y evaluación de competencias en educación superior. Madrid: Narcea.

Corona, J. y Sastre, E. (2018). Orientación profesional y formación dual. Hacia un modelo integrado para el empleo juvenil. Madrid: Instituto de la Empresa Familiar.

España, Ley 11/2020, de 30 de diciembre, de Presupuestos Generales del Estado para el año 2021. BOE, 31 de diciembre de 2020, núm. 341, p. 125958-126732

España, Real Decreto 1393/2007, de 29 de octubre, por el que se establece la ordenación de las enseñanzas universitarias oficiales. BOE, 30 de octubre de 2007, núm. 260, p. 44037-44048

España, Real Decreto Legislativo 2/2015, de 23 de octubre, por el que se aprueba el texto refundido de la Ley del Estatuto de los Trabajadores. BOE, 24 de octubre de 2015, núm. 255, p. 100224-100308

España, Resolución del 20 de octubre de 2020, del Director de Unibasq -Agencia de Calidad del Sistema Universitario Vasco, por la que se da publicidad al Protocolo para la obtención del reconocimiento de formación dual para títulos universitarios oficiales de Grado y Máster, aprobado por la Comisión Asesora de Unibasq el 16 de octubre de 2020 .

Loades, R. (2005). The future of graduate Management education in the context of Bolonia. Accord Graduate Management. Admission Council.

Villa, A. y Poblete, M. (2007). Aprendizaje basado en competencias: una propuesta para la evaluación de las competencias genéricas. Bilbao: Editorial Mensajero, S.A.U. 